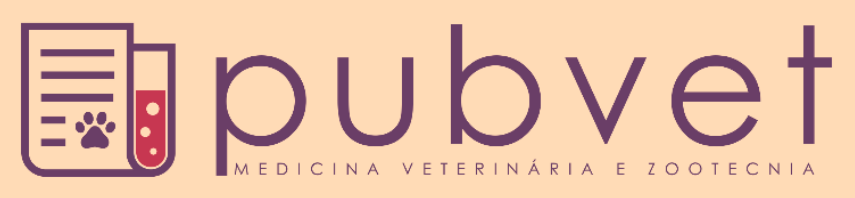

https://doi.org/10.31533/pubvet.v12n11a210.1-8

\title{
Indução de novilhas para protocolo de inseminação artificial em tempo fixo: Revisão
}

\author{
Ricardo Brognoli de Oliveira ${ }^{1}$, Baltazar Alves da Silva Junior ${ }^{2 *}$, Thiago Henrique \\ Cassiano Cavalcanti ${ }^{30}$
}

${ }^{I}$ Acadêmico de Medicina Veterinária da Faculdade Anhanguera de Dourados, Dourados - MS Brasil.

${ }^{2}$ Professor Me. Faculdade Anhanguera de Dourados, Dourados - MS Brasil.

${ }^{3}$ Medico Veterinário Reprovet, Dourados - MS Brasil.

*Autor para correspondência. E-mail: bjr_vet@hotmail.com

RESUMO. Este trabalho teve como objetivo descrever alguns conceitos importantes referentes à fisiologia reprodutiva, do controle farmacológico do ciclo estral, apresentando diferentes protocolos para a inseminação artificial em tempo fixo (IATF). A IATF se tornou uma das principais biotecnologias reprodutivas de importância econômica na produção de bovinos de maneira global. Os diferentes fármacos existentes atualmente no mercado permitem que se realize a sincronização do ciclo estral em novilhas com o intuito de maximizar os índices reprodutivos. Na literatura atual, inúmeros protocolos são citados capazes de induzir a ovulação para a IATF, fazendo necessário que se conheça de maneira ampla e aprofundada esses protocolos, afim de que seja escolhido o melhor para determinada situação e objetivo.

Palavras chave: ciclo estral, fisiologia reprodutiva, sincronização

\section{Induction of heifers for artificial insemination protocol at fixed time: Review}

\begin{abstract}
The present work aimed to describe some important concepts regarding the reproductive physiology of pharmacological control of the estrous cycle, presenting different protocols for IATF. IATF has become one of the leading reproductive biotechnologies of economic importance in cattle production globally. The different drugs currently available on the market allow synchronization of the estrous cycle in heifers with the aim of maximizing reproductive rates. In the current literature, numerous protocols are cited capable of inducing ovulation for IATF, making it necessary to know these protocols in a broad and deep way, in order to select the best for a given situation and objective.
\end{abstract}

Keywords: estrous cycle, reproductive physiology, synchronization

\section{Inducción de novillas para el protocolo de inseminación a tiempo fijo: Revisión}

RESUMEN. El presente trabajo tiene como objetivo describir algunos conceptos importantes relacionados con la fisiología reproductiva, control farmacológico del ciclo estral, mostrando diferentes protocolos de Inseminación artificial a tiempo fijo (IATF). La IATF se ha convertido en uno de las principales biotecnologías reproductivas de importancia económica en ganadería a nivel mundial. Los diferentes fármacos existentes actualmente en el mercado permiten que se realice la sincronización del ciclo estral en novillas para maximizar los índices reproductivos. En la literatura actual, muchos protocolos son mencionados como capaces de inducir la ovulación para IATF, creando la 
necesidad de conocer amplia y profundamente esos protocolos, a fin de que sea escogido el mejor para determinada situación y objetivo.

Palabras clave: ciclo estral, fisiología reproductiva, sincronización

\section{Introdução}

Um dos pontos críticos quando falamos em eficiência reprodutiva é a idade ao primeiro parto. É o parâmetro mais confiável quando o objetivo é maximizar a eficiência reprodutiva, devido ao fato de expressar o melhor índice para a avaliação de fertilidade das novilhas (Sá Filho et al., 2011).

Na maioria das propriedades de cria do país, o sistema empregado utiliza períodos restritos de cobertura, o que requer novilhas que atinjam a puberdade mais cedo para obter um alto desempenho reprodutivo nas concepções subsequentes. Baseado nisso, estudos demonstram que novilhas que possuem um corpo lúteo no início da estação de monta apresentam maior taxa de prenhez quando são submetidas a Inseminação Artificial em Tempo Fixo (IATF), quando comparadas às novilhas que não estão cíclicas no início da estação de monta (Funston et al., 2012). A IATF foi desenvolvida com o intuito de eliminar os problemas da inseminação artificial convencional como, por exemplo, as falhas na observação do cio. Esse procedimento possibilita a inseminação de um maior número de animais em dia e horário desejados, diminuindo o tempo do anestro pós-parto (Fernandes \& Matos, 2016).

Nos últimos anos foram desenvolvidos diversos protocolos hormonais com o objetivo de induzir a puberdade precoce em novilhas, o que consequentemente aumenta os índices reprodutivos do rebanho. A maioria dos protocolos hormonais utilizam a progesterona e estrógeno, hormônios responsáveis pela indução da ovulação nas fêmeas e por alterar o funcionamento do eixo hipotálamo-hipofisário (Santos et al., 2018).

Este estudo teve como objetivo geral descrever os diferentes protocolos hormonais utilizados em indução de novilhas por meio da IATF. Seus objetivos específicos são apresentar os diferentes protocolos hormonais; discutir as vantagens e desvantagens de cada protocolo; apresentar qual o melhor protocolo de IATF para a indução de novilhas baseado em literatura já existente.

\section{Inseminação Artificial em Tempo Fixo (IATF)}

Diversos fatores podem influenciar a idade à puberdade em novilhas de corte, sendo que quando não se alcançada logo no início da estação de monta, compromete de maneira direta a eficiência reprodutiva (Pereira et al., 2010). Desse modo, os tratamentos que são direcionados à indução da puberdade em novilhas têm o objetivo de aumentar a eficiência reprodutiva, permitindo que as fêmeas completem o primeiro estro subfértil antes do início da estação de monta. A novilhas que são cobertas nesse período conseguem parir no início estação de produção e os terneiros são mais pesados ao desmame, tendo um maior tempo para recuperar as suas reservas corporais no período de pós-parto (Bragança et al., 2013).

O grande obstáculo quando se trata de inseminação artificial convencional no Brasil, consiste na detecção de estro (Pereira et al., 2010).

$\mathrm{O}$ uso da IATF traz diversas vantagens por permitir que um maior número de animais seja inseminados em um menor período de tempo, já que com esse método as vacas têm a ovulação induzida, podendo ser realizada em data e horário específico, de acordo com a disponibilidade do produtor (Inforzato et al., 2008).

Como a ovulação é induzida, o produtor não necessita se preocupar com a observação do cio no rebanho, o que gera uma economia na mão de obra, bem como concentra o nascimento dos bezerros. Além dessas vantagens, é notável que quando se utiliza a IATF há um aumento na produção de leite e de bezerros, aumentando a eficiência reprodutiva e diminuindo o intervalo entre partos (Baruselli et al., 2012). Entretanto, existe uma desvantagem da IATF quando comparado com a convencional, que é a viabilidade econômica, onde em algumas situações o custo benefício pode não ser viável, devido aos custos dos fármacos utilizados nos protocolos. Todavia, esse custo se torna alto apenas ao introduzir esse método na propriedade, posteriormente há uma redução lenta no custo desses fármacos o que gera menores custos para a sincronização da ovulação (Baruselli et al., 2012).

Em propriedades destinadas à produção de touros e/ou matrizes é notável o retorno financeiro pelo uso da IATF. E para que bons resultados sejam alcançados é imprescindível a seleção de vacas no intuito de selecionar as melhores matrizes no rebanho (Inforzato et al., 2008). 
Nos bovinos, o ciclo sexual não está ligado à estação do ano desde que as condições de nutrição e adaptação ambiental seja favorável. Assim, as vacas são caracterizadas como poliéstrica não sazonal. A ativação, crescimento e maturação folicular são chamados de processo de foliculogênese, tendo início com a formação dos folículos durante a vida fetal, e isso significa que ao nascer as crias já tem determinado a quantidade de folículos primordiais em suas gônadas. A maior parte desses folículos são degenerados devido à atresia folicular, onde apenas alguns folículos são maturados para a ovulação (Sá Filho et al., 2011).

\section{Ciclo estral}

O ciclo sexual em fêmeas bovinas é caracterizado por quatro fases, sendo proestro, estro, metaestro e diestro. O proestro é onde ocorre a maturação folicular, o estro é marcado pela manifestação do cio, o metaestro é marcado pelo processo de ovulação e formação do corpo lúteo e por fim, o diestro é marcado pela atividade do corpo lúteo e secreção de progesterona (Sá Filho et al., 2011). O proestro é a fase que antecede o cio onde alguns sinais podem ser percebidos como inquietação, cauda erguida, urina constante, vulva edemaciada e brilhante, diminuição do apetite, vocalização, estresse, liberação de muco viscoso e brilhante. Além de todos esses sinais, o que se torna mais característico e fácil de ser visualizado é a tentativa da vaca de tentar montar nas outras. Essa fase tem dura em média de 2 a 3 dias, terminando quando a fêmea começa a aceitar o macho (Sá Filho et al., 2011). Já o estro é a fase onde a fêmea passa a apresentar os sinais de receptividade sexual seguida da ovulação e tem uma duração média de 21 dias. Quando essa fase inicia, a vaca aceita a monta do macho apenas no período do cio, que dura em média de 11 a 18 horas, e após o término, não aceita mais a monta tanto de outras fêmeas quanto por touros e rufiões (Sá Filho et al., 2011). O metaestro não apresenta características tão perceptíveis quanto as outras fases, durando em média de 2 a 4 dias. Seu término ocorre quando o corpo lúteo começa a sintetizar a própria progesterona e é nesse fase onde ocorre a ovulação (Pereira et al., 2010). Por último, o diestro que é a fase onde o corpo lúteo já está secretando progesterona de maneira constante. Nessa fase há ação do esteroide, onde o endométrio passa a ficar mais largo e com maior atividade glandular, a cérvix regride, a musculatura do genital relaxa e também ocorre uma diminuição da vascularização e hipotrofia do epitélio vaginal. A duração dessa fase é de 14 dias e só termina quando o corpo lúteo sofre o processo de lise; o que leva a um novo ciclo fisiológico (Sá Filho et al., 2011).

\section{Métodos de sincronização}

Os fatos de toda a história dessa biotécnica associado ao emprego da inseminação artificial em bovinos, provam que as descobertas da ciência podem serem aplicadas ao avanço das técnicas utilizadas para o manejo reprodutivo nessa espécie. A primeira sincronização realizada foi relatada no ano de 1948 e foi a partir de então que as pesquisam começaram a focar em programas e produtos de sincronização para cada vez mais obter avanços relacionados a essa técnica. A sincronização é implantada objetivando a lucratividade pelo aumento da taxa de serviço, minimizando também os problemas de observação de cio (Bragança et al., 2013).

A sincronização da ovulação é uma ferramenta essencial em relação ao manejo reprodutivo do rebanho para a realização da IATF. Na literatura atual existem diferentes técnicas hormonais, permitindo o controle do desenvolvimento folicular e da ovulação em rebanhos bovinos. $\mathrm{O}$ ciclo estral é resultante da interação coordenada dos tecidos do sistema nervoso central, hipotálamo-hipófise, ovário e útero (Fernandes \& Matos, 2016).

Os principais hormônios responsáveis pela comunicação entre os órgãos são os hormônios GnRH (hipotálamo), hormônio luteizinante (LH) e hormônio folículo estimulante (FSH) (hipófise), estradiol e progesterona (ovários) e prostaglandina $\mathrm{F}_{2} \alpha$ (útero). Os protocolos são baseados basicamente em duas estratégias, onde a primeira induz a regressão do corpo lúteo, com o intuito de sincronizar o início da fase folicular; e a segunda consiste em alongar a fase luteínica de maneira artificial com a aplicação exógena de progesterona, fazendo que com que os animais entrem na fase folicular ao mesmo tempo (Mattos et al., 2012). Existem duas estratégias onde os protocolos de sincronização são baseados: a primeira é a indução da regressão do corpo lúteo de maneira que todas as vacas entrem na fase folicular ao mesmo para que mantenham a sincronização durante o estro. A segunda consiste em alongar a fase luteína de maneira artificial pela administração de uma fonte exógena de progesterona fazendo com que todos as fêmeas entrem na fase folicular ao mesmo tempo 
(Fernandes \& Matos, 2016). Quando se trata do controle do ciclo estral é possível pensarmos em duas vertentes distintas: a sincronização e a indução de cio e/ou ovulação. A sincronização é definida como o ato de antecipar ou prorrogar o ciclo estral em animais que estão ciclando de maneira normal, enquanto a indução é o ato de induzir o cio em animais que estejam em anestro, através de hormônios ou manejo específico para tal. Em algumas situações, é possível que se realize protocolos que apresentem efeito de sincronização e indução de cios de modo simultâneo (Fernandes \& Matos, 2016; Mattos et al., 2012).

A sincronização e indução do estro em novilhas é uma ferramenta importante que auxilia na repetição de crias em novilhas. A sincronização do estro e a indução da ovulação, tanto em novilhas cíclicas como nas ainda acíclicas, podem fazer parte do manejo da reprodução, além do fato de que possibilitam a utilização da inseminação artificial a tempo fixo. Todavia, esses recursos só são indicados quando se atende a outros requisitos, principalmente aqueles associados ao manejo nutricional (Azeredo et al., 2007).

\section{Fármacos utilizados nos protocolos hormonais}

O sistema reprodutivo, tanto do macho quanto da fêmea é controlado pela ação de diversos hormônios. Nas fêmeas bovinas existem maiores estudos acerca das substâncias hormonais, que são sintetizadas por diferentes órgãos, atuando sobre o sistema reprodutivo, em um esquema complexo de inter-relações, e desse modo a escolha das substâncias a serem utilizados requer amplo conhecimento (Bragança et al., 2013).

\section{Progesterona e estrógeno.}

$\mathrm{O}$ uso da progesterona aumenta a eficiência reprodutiva no rebanho, aumenta as taxas de cio bem como de concepção, estando ou não associados a indução da ovulação. O objetivo da utilização da progesterona (P4) é a viabilização da manipulação exógena da atividade ovariana, bem como do controle folicular, permitindo a sincronização do cio e a implantação de um programa de IATF. Em sistemas extensivos de criação de rebanhos de bovinos de corte foi observado anestro em aproximadamente 50\% das vacas no início da estação de monta (Godoy et al., 2010). A administração de implante de $\mathrm{P} 4$ permite a regressão do corpo lúteo, já estando este iniciado, e um feedback negativo na liberação de LH pelo hipotálamo. Após a remoção do mesmo, ocorre o crescimento folicular, o cio e ovulação entre 2 e 8 dias após a remoção (Baruselli et al., 2012).

A maioria dos protocolos hormonais utilizam a associação de P4 com algum luteolítico, sendo entre eles o mais usado o estrógeno, com o objetivo de sincronizar o aparecimento de uma onda folicular e a ovulação. A associação da P4 com o estrógeno resulta em uma nova onda folicular de 4 a 5 dias após a aplicação (Baruselli et al., 2012). O estrógeno tem ação luteolítica e em contrapartida a $\mathrm{P} 4$ inibe o desenvolvimento do corpo lúteo ou previne a ovulação. $\mathrm{O}$ estrógeno apresenta uma importante função no controle fisiológico das vacas, de maneira a induzir o pico pré ovulatório de LH e ovulação. Os efeitos do estradiol auxiliam a ação da P4, justificando o seu uso associado, otimizando a precisão da sincronização (Godoy et al., 2010).

\section{Gonadotrofina coriônica equina (eCG)}

A Gonadotrofina Coriônica Equina consiste em um hormônio de caráter glicoproteico que é secretado pelos cálices endometriais de éguas gestantes. Possui ação parecida com a do FSH e LH, sendo que o uso é indicado para rebanhos com baixos índices de ciclicidade. $\mathrm{O}$ uso de eCG se tornou essencial para programas de IATF, tanto para a indução do cio como para a ovulação (Mello et al., 2014).

Em pesquisas realizadas foi possível notar que a associação de eCG no momento da retirada do dispositivo de liberação de P4 e de Gonadotrofina Coriônica Humana (hCG), indutor da ovulação em 48 horas, proporcionou melhor taxa de concepção em relação a outros protocolos (Mello et al., 2014).

O eCG tem meia vida em torno de 46 horas, sendo o único com capacidade de exercer atividade folículo estimulante e luteinizante ao mesmo tempo, o que auxilia nas concentrações de $\mathrm{P} 4$, o resultando no aumento das taxas de prenhez (Souza et al., 2015). O eCG auxilia ainda na taxa de crescimento dos folículos, acarretando um folículo pré ovulatório maior, e consequentemente um maior corpo lúteo. Melhores índices de prenhez são atingidos em rebanhos onde se utiliza o eCG em vacas que se encontram em anestro pósparto e/ou com condição nutricional diminuída. Há ainda relatos na literatura onde a eCG é utilizada após a IA com o objetivo de aumentar a concentração plasmática de $\mathrm{P} 4$, obtendo melhores taxas de concepção (Mello et al., 2014). 


\section{Prostaglandina $F_{2} \alpha$}

Sendo o fármaco de maior utilização para a sincronização de cio, a prostaglandina $F_{2} \alpha$, tem um papel essencial em programas de IATF. Em contrapartida, o estro após o tratamento é distribuído no decorrer de seis dias, sendo influenciado pela resposta do corpo lúteo, bem como pelo estágio do desenvolvimento que o folículo dominante de encontra (Uribe-Velásquez et al., 2011). Moreno et al. (1986) avaliaram vacas Bos indicus para detectar a presença de corpo lúteo e as trataram com $\mathrm{PGF}_{2} \alpha$. Foi observado de 80 a $100 \%$ de luteólise. No entanto, apenas 47 a $60 \%$ foram detectadas em estro após o tratamento. Essa alta na variabilidade de respostas ao tratamento com $\mathrm{PGF}_{2} \alpha$ e o fato de animais criados a pasto em condições tropicais apresentarem alta incidência de anestro comprometem de forma direta a eficácia do tratamento, sendo necessário que se faça o emprego de outro meio para que haja a sincronização do desenvolvimento luteínico e folicular com o intuito de controlar o crescimento dos folículos e da ovulação. Isso permite que haja o emprego da IATF sem que exista a necessidade da detecção do cio.

\section{Hormônio liberador de gonadotrofinas (GnRH)}

$\mathrm{O}$ GnRH tem função de induzir o pico pré ovulatório de LH e a ovulação de um folículo maduro bem como sua luteinização. Esse hormônio tem efeitos variáveis na função do corpo lúteo, sendo influenciado pelo momento do ciclo no qual o tratamento é iniciado. Há estudos que relatam que a associação de progesterona exógena com GnRH e prostaglandina, melhoraram a taxas de prenhez em novilhas (Bragança et al., 2013). O GnRH é sintetizado e secretados em dois centros diferentes do hipotálamo, sendo um o centro de secreção tônica, secretando de forma contínua e o outro centro controlador da onda pré-ovulatória, que liberam grandes quantidades de maneira única, por meio do sistema porta hipotalâmicohipofisário, de modo a atingir a hipófise anterior e fazendo a estimulação da liberação de FSH e LH, que chegam aos ovários pela circulação sanguínea especificamente nos folículos, onde o FSH tem função no desenvolvimentos dos mesmos (Furtado et al., 2011; Martemucci \& D’Alessandro, 2011).

\section{Principais protocolos utilizados na IATF}

Os protocolos de sincronização de IATF tem o objetivo de induzir uma nova onda de crescimento folicular, controlar a duração do crescimento folicular até a pré ovulação, e induzir a ovulação sincronizada em todas as fêmeas de maneira simultânea (Crepaldi, 2009).

Com o objetivo de maximizar a eficiência na taxa de prenhez em programas de IATF, diversos protocolos são desenvolvidos com o objetivo de sincronizar o crescimento folicular e a ovulação em novilhas. Para obter resultados positivos alguns fatores devem ser levados em consideração sendo desde a seleção das novilhas, qual hormônio utilizar, qual protocolo escolher, qualidade do sêmen, dentro outros. Existem diversos protocolos capazes de sincronizar a ovulação para realização de IATF onde a escolhe do protocolo a ser utilizado varia de acordo com a avaliação técnica das condições das novilhas a serem inseminados (Crepaldi, 2009).

O primeiro protocolo é com o uso de eCG préovulatório. O uso desse protocolo é baseado na estimulação do crescimento do folículo préovulatório, com o intuito de que o mesmo se torne um corpo lúteo com uma maior produção de progesterona. É realizada aplicação de estradiol e colocado o implante de $\mathrm{PGF}_{2} \alpha$. Nove dias após é retirado o implante, é feita a aplicação de uma dose de eCG e 48 horas depois é realizada a IA, iniciando o D0 (Figura 1). Com a utilização desse protocolo o diagnóstico de prenhez pode ser realizado no D28 (Machado, 2012).

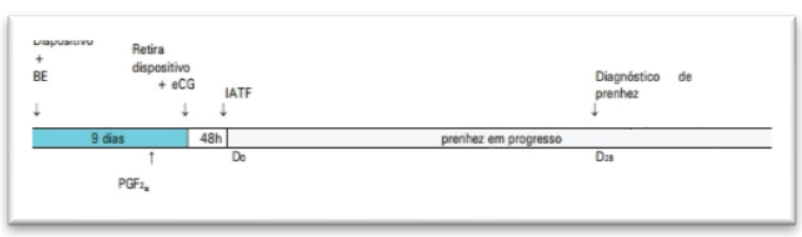

Figura 1. Protocolo para aumentar a produção de progesterona com utilização de eCG antes da ovulação. Fonte: Machado (2012).

Outro protocolo citado por Machado (2012) é o que utiliza como base a aplicação de GnRH, cinco dias após a realização da IA, e de gonadotrofina coriônica humana (hCG) 12 dias após a inseminação. Esse protocolo auxilia no processo da ovulação de um folículo em desenvolvimento, ou seja, a primeira onda folicular, e como consequência a formação de um corpo lúteo acessório, bem como o recrutamento de novos folículos, sendo este responsável pela segunda onda folicular. Pode ocorrer ainda uma terceira onda folicular entre os dias 14 e 15, como consequência da aplicação de hCG e da presença da segunda onda (Figura 2). 
Tanto o primeiro quanto o segundo protocolo foram testados em rebanhos da raça Nelore na Embrapa Pecuária Sudeste. Nos animais onde foi administrado a eCG, notou-se uma manutenção na concentração de progesterona na fase luteínica e um aumento de cerca de $13 \%$ na taxa de prenhez (Machado, 2012).

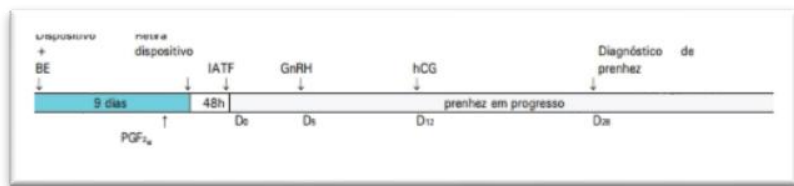

Figura 2. Protocolo para reduzir as concentrações de estradiol e aumentar a produção de progesterona com utilização de GnRH e hCG após a ovulação. Fonte: Machado (2012).

Protocolos que tem como base estrógeno e progestágeno estão sendo comumente utilizados em programas de IATF em bovinos. A técnica é caracterizada pela inserção de um implante contendo progestágeno associado à administração de benzoato de estradiol no D0, objetivando o início de uma onda folicular (Furtado et al., 2011).

Após a retirada do implante dos dias 7, 8 ou 9 é feita a administração de PGF2 $\alpha$ com o intuito de induzir a luteólise bem como minimizar o nível de P4 circulante (Figura 3). Após 24 horas é realizado novamente a administração de benzoato de estradiol para que a ovulação seja sincronizada, devido ao fato da indução de um pico de LH a partir de um feedback positivo pré - ovulatório ao GnRH, sendo a IA realizada de 30 a 36 horas após a aplicação (Furtado et al., 2011).

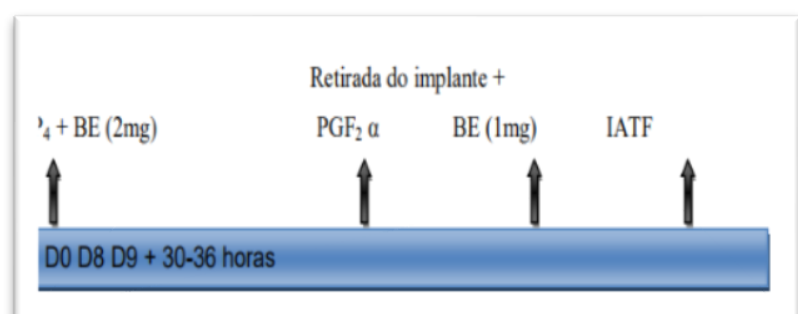

Figura 3. Associação entre Benzoato de Estradiol (BE) e Progestágeno (P4). Fonte: Furtado et al. (2011).

De acordo com Furtado et al. (2011) existe ainda um protocolo onde é utilizado a associação de cipionato de estradiol (ECP) e P4 (Figura 4). Essa associação acaba gerando atresia folicular de todos os folículos existentes nos ovários, acarretando a necessidade de uma nova onda folicular já que o ECP possui meia vida muito prolongada, cerda de 10 a 12 dias. Pelo fato da meia vida ser mais longa quando comparado ao benzoato de estradiol por exemplo, o ECP faz com que ocorra um atraso de início de onda nas novilhas e vacas, prejudicando desse modo os resultados de prenhez. Isso ocorre devido ao fato de que grande parte das fêmeas não possui um folículo pré-ovulatório no momento da IATF.

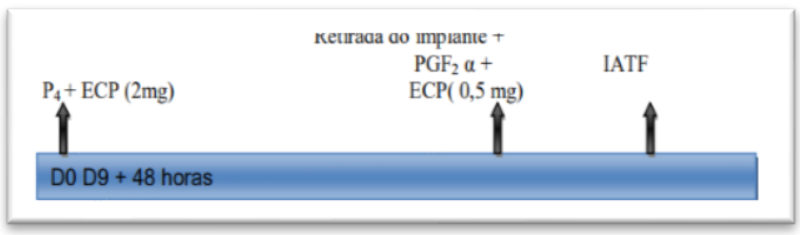

Figura 4. Protocolo de IATF com uso de progesterona e cipionato de estradiol. Fonte: Furtado et al. (2011).

Um outro protocolo que existe e quem vem sendo muito utilizado é o protocolo de Ovsynch (Figura 5), caracterizado pela combinação da administração de três hormônios onde cada aplicação possui um objetivo diferente. A primeira administração é de GnRH onde tem o intuito de estimular a ovulação ou regressão do folículo dominante e de a emergência de uma nova onde folicular já que induz uma grande síntese de LH. (Azevedo \& Simões, 2014).

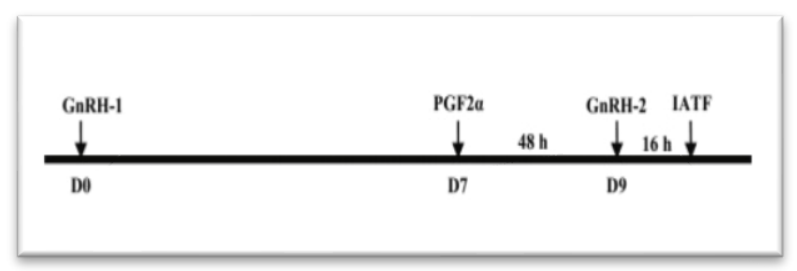

Figura 5. Representação esquemática do protocolo Ovsynch. Fonte: Azevedo and Simões (2014).

A segunda é a administração é de $\mathrm{PGF}_{2} \alpha 48$ horas antes da segunda aplicação de GnRH, denominada de $\mathrm{GnRH}-2$, permitindo que o terceiro objetivo seja realizado, que é a indução do pico de LH que irá resultar na ovulação do folículo dominante. A inseminação é realizada de 16 horas após a administração de GnRH-2 (Azevedo \& Simões, 2014).

Com o protocolo de Ovsynch, observou-se que a sincronização do cio e ovulação elevam seus índices em mais de 90\%, devido a segunda administração de $\mathrm{GnRH}$ após 48 horas da aplicação de $\mathrm{PGF}_{2} \alpha$. Há pouca variação da taxa de fertilidade devido à inseminação 16 horas depois da segunda aplicação de GnRH, quando comparados a observação de cio (Furtado et al., 2011). 


\section{Considerações finais}

A indução da ovulação representa na atualidade uma excelente alternativa para que se amplie o uso da IATF em novilhas já que tem sido relatada taxas significativas de prenhez com o uso da técnica. Todavia, é necessário que todos os fatores de variação sejam levados em consideração no momento da escolha do protocolo adequado e as condições de aplicação para que se alcance resultados satisfatórios. Portanto é cabível que o protocolo escolhido atenda todas as necessidades do produtor, devido aos vários protocolos existentes no mercado bem como seus prós e contras.

\section{Referências}

Azeredo, D. M., Rocha, D. C., Jobim, M. I. M., Mattos, R. C., \& Gregory, R. M. 2007. Efeito da sincronização e indução de estros em novilhas sobre a prenhez e o índice de repetição de crias na segunda estação reprodutiva. Ciência Rural, 37(1), 201-2015.

Azevedo, C., \& Simões, J. 2014. O protocolo hormonal ovsynch e suas modificações em vacas leiteiras de alta produção: uma revisão. Archivos de Zootecnia, 63(241), 173-187.

Baruselli, P. S., Sales, J. N. S., Sala, R. V., Vieira, L. M., \& Sá Filho, M. F. 2012. History, evolution and perspectives of timed artificial insemination programs in Brazil. Animal Reproduction, 9(3), 139-152.

Bragança, J. F. M., Ferreira, R., Loguercio, R., Camponogara, R., Loguercio, L., Oliveira, J. F., \& Gonçalves, P. B. D. 2013. Programa de sincronização/indução de estro e ovulação para novilhas de corte com 12 a 14 meses de idade. Revista Portuguesa de Ciências Veterinárias, 108, 137-142.

Crepaldi, G. A. 2009. Eficácia de diferentes protocolos de indução da ovulação e de intervalos de inseminação em vacas de corte submetidas à IATF. Master of Science, Universidade de São Paulo, São Paulo, Brasil.

Fernandes, I. C., \& Matos, A. T. 2016. Indução hormonal em novilhas nulíparas com posterior protocolo de inseminação artificial em tempo fixo (IATF) como alternativa para maximizar a eficiência reprodutiva. Revista Eletrônica da Faculdade de Ciências Exatas e Agrárias Produção/Construção e Tecnologia, 5(9), 110.

Funston, R. N., Musgrave, J. A., Meyer, T. L., \& Larson, D. M. 2012. Effect of calving distribution on beef cattle progeny performance. Journal of Animal Science, 90(13), 5118-5121.

Furtado, D. A., Tozzetti, D. S., Avanza, M. F. B., \& Dias, L. G. G. G. 2011. Inseminação artificial em tempo fixo em bovinos de corte. Revista Científica Eletrônica de Medicina Veterinária, 16, 1-25.

Godoy, C. R., Silva, E. F. P., \& Paula, A. P. 2010. Inseminação artificial em tempo fixo (IATF) em bovinos de corte. PUBVET, 4, Art. 802808.

Inforzato, G. R., Santos, W. R. M., Climeni, B. S. O., Dellalibera, F. L., \& Filadelpho, A. L. 2008. Emprego de IATF (Inseminação Artificial em Tempo Fixo) como alternativa na reprodução da pecuária de corte. Revista Científica Eletrônica de Medicina Veterinária, 11, 1-8.

Machado, R. 2012. Protocolos para otimizar a fertilidade de vacas de corte e leite. Embrapa Pecuária Sudeste, 1, 1-7.

Martemucci, G., \& D'Alessandro, A. G. 2011. Induction/synchronization of oestrus and ovulation in dairy goats with different short term treatments and fixed time intrauterine or exocervical insemination system. Animal Reproduction Science, 126(3-4), 187-194.

Mattos, F. C. S. Z., Bastos, M. R., Canavassi, A. M. O., Lemes, A. P., Paduan, M., Susin, I. \& Sartori, R. 2012. Influence of feed intake on plasma concentrations of insulin and progesterone and expression of liver enzymes involved in metabolism of progesterone in sheep. Official Journal of the Brazilian College of Animal Reproduction, 9, 608-608.

Mello, R. R. C., Ferreira, J. C., Mello, M. R. B., \& Palhano, H. B. 2014. Utilização da gonadotrofina coriônica equina (eCG) em protocolos de sincronização da ovulação para IATF em bovinos: revisão. Revista Brasileira de Reprodução Animal, 38, 129-134.

Moreno, I. Y. D., Galina, C. S., Escobar, F. J., Ramírez, B., \& Navarro-Fierro, R. 1986. Evaluation of the lytic response of prostaglandin F2 alpha in Zebu cattle based on serum progesterone. Theriogenology, 25(3), 413-421.

Pereira, E. S., Pimentel, P. G., Queiroz, A. C., \& Mizubuti, I. Y. 2010. Novilhas leiteiras (Vol. 1). Fortaleza, Ceará: Graphiti Gráfica e Editora Ltda. 
Sá Filho, M. F., Baldrighi, J. M., Sales, J. N. S., Crepaldi, G. A., Carvalho, J. B. P., Bó, G. A., $\&$ Baruselli, P. S. 2011. Induction of ovarian follicular wave emergence and ovulation in progestin-based timed artificial insemination protocols for Bos indicus cattle. Animal Reproduction Science, 129(3-4), 132-139.

Santos, M. H., Ferraz Júnior, M. V. C., Polizel, D. M., Barroso, J. P. R., Miszura, A. A., Martins, A. S. \& Pires, A. V. 2018. Decreasing from 9 to 7 days the permanence of progesterone inserts make possible their use up to 5 folds in suckled Nellore cows. Theriogenology, 111, 56-61.

Souza, A. L. B., Kozicki, L. E., Pereira, J. F. S., Segui, M. S., Weiss, R. R., \& Bertol, M. A. F. 2015. Eficiência da gonadotrofina coriônica equina (eCG) e do desmame temporário (DT) em protocolos para a inseminação artificial em tempo fixo (IATF) em vacas nelore, previamente tratadas com progesterona $(\mathrm{P} 4) \mathrm{e}$ benzoato de estradiol (BE). Archives of Veterinary Science, 20(1), 22-29.

Uribe-Velásquez, L. F., Souza, M. I. L., \& Osorio, J. H. 2011. Efeito do tempo da aplicação de prostaglandina na resposta folicular em ovelhas durante o ciclo estral. 985-991, 40(5), 985-991.

Recebido: 6 Agosto 2018 .

Aprovado: 1 Setembro 2018.

Publicado: 9 Novembro 2018.

Licenciamento: Este artigo é publicado na modalidade Acesso Aberto sob a licença Creative Commons Atribuição 4.0 (CC-BY 4.0), a qual permite uso irrestrito, distribuição, reprodução em qualquer meio, desde que o autor e a fonte sejam devidamente creditados. 